\title{
BMP-2 and BMP-4 signalling in the developing spinal cord of human and rat embryos
}

\author{
A. Namm ${ }^{1,2}$, A. Arend ${ }^{1}$, M. Aunapuu ${ }^{1,2}$ \\ ${ }^{1}$ Department of Anatomy, University of Tartu, Tartu, Estonia \\ ${ }^{2}$ Department of Morphology, Estonian University of Life Sciences, Tartu, Estonia
}

[Received 25 November 2014; Accepted 18 December 2014]

\begin{abstract}
Bone morphogenetic proteins (BMPs) are multifunctional growth factors implicated in multiple biological events. Studies on mice, chickens and other experimental animals have shown that BMP signalling plays critical role in embryonic development, in particular in the neural patterning. In our study we comparatively evaluated $B M P-2$ and BMP-4 protein expression in the developing spinal cord of human and rat embryos. The human and rat embryos of Carnegie stages 14, 18 and 20 were embedded in paraffin and cut serially in transversal direction. BMP-2 and BMP-4 were detected by immunohistochemical staining. Spatial and temporal expression pattern of BMP-s during early stages of spinal cord development was similar in human and rat embryos. Higher expression of BMP-s was seen in the dorsal and lower expression in the ventral part of the developing spinal cord both in human and rat embryos. However, temporal difference in the expression of BMPs in the non-neural ectoderm between human and rat embryos was noted. Staining of BMP-s in the non-neural ectoderm adjacent to the developing spinal cord in the human embryos seemed to have a tendency to decrease from earlier to later developmental stages, while in rat embryos there was an opposite tendency. (Folia Morphol 2015; 74, 3: 359-364)
\end{abstract}

Key words: BMP-2 and BMP-4, human and rat embryos, spinal cord development

\section{INTRODUCTION}

Bone morphogenetic proteins (BMPs) are signalling molecules that act at distance to control cell fate in the developing embryos. Although BMPs were originally identified by an ability to induce the formation of cartilage and bone, it is now generally accepted that BMPs are among the key regulators in the embryonic development guiding many critical steps in the formation of various cells, tissues and organs $[4,10]$. BMPs in conjunction with other important regulatory factors like Sonic Hedgehog (SHH), WNT and FGF family members are believed to coordinate neural tube development and cellular proliferation $[10,13,15]$. Experimental data from studies mainly on mice and chickens have provided evidence that BMPs are implicated in the patterning of the developing central nervous system (CNS) [3, 5], in particular BMPs have been shown to regulate dorso-ventral patterning of the vertebrate spinal cord $[14,16]$. Although BMP activity must be inhibited to allow initial neural fate determination, it is clear that BMP signalling positively regulates CNS development at later stages [13]. Once the neural tissue is established, BMP-2 and BMP-4 signalling has a positive influence on the regulation of dorsal neural cell type formation [10]. Several studies have demonstrated that formation and regionalisation of spinal cord is highly dependent on activity of the roof plate sig-

Address for correspondence: Dr A. Namm, Department of Anatomy, University of Tartu, Ravila 19, Biomedicum, 50411 Tartu, Estonia, tel: +372 7374 257, fax: +372 7374 252, e-mail: aimar.namm@ut.ee 
nalling activity. As the caudal neural tube closes the roof plate progenitors differentiate into mature roof plate cells, which occupy the dorsal-midline region and act as an organising centre controlling numerous aspects of dorsal spinal cord development [1, 2]. In studies both on mice and chickens expression of BMPs in the roof plate during neural tube development at the time of dorsal neuronal cells formation has been clearly shown $[7,8]$.

In our previous study on human embryos we found strong BMP-2 and BMP-4 protein expression in the dorsal part of the developing spinal cord, including also the roof plate region [11]. In order to see if this temporal and spatial BMP-2 and BMP-4 protein expression pattern found in the forming nervous system of human embryos is equally similar to the situation in embryos of other mammals we aimed in this study to compare the BMP-2 and BMP-4 expression in the developing spinal cord of human and rat embryos throughout early stages of development.

\section{MATERIALS AND METHODS}

\section{Obtaining embryos and section preparation}

Twenty two human embryos of Carnegie stages (CS) 14, 18 and 20 were obtained by medical abortions. The study was approved by the Ethics Review Committee on Human Research of the University of Tartu. 22 Sprague Dawley strain rat embryos of CS 14,18 and 20 were used in this study (rat embryos were obtained from the Umea University, Sweden). The human and rat embryos were fixed in $4 \%$ paraformaldehyde and embedded in paraffin according to standard methods. Tissue blocks were serially cut in transversal direction and mounted on glass slides.

Embryos were classified by CS [12]. The developmental stages and the number of embryos used in this study are presented in Table 1. This table also presents an approximate comparison of human and rat embryos based upon Carnegie staging.

\section{Immunohistochemistry}

Three- $\mu \mathrm{m}$ thick paraffin sections mounted on poly-L-lysine coated slides were deparaffinised and rehydrated. Peroxidase activity was blocked by $0.6 \% \mathrm{H}_{2} \mathrm{O}_{2}$ in methanol (Merck, Darmstadt, Germany). Then the sections were washed in tap water and in phosphate buffered saline (PBS) $(\mathrm{pH}=7.4)$ for $10 \mathrm{~min}$, treated with normal $1.5 \%$ goat serum (Gibco, Invitrogen Corporation, USA) for $20 \mathrm{~min}$ at room temperature
Table 1. Number of studied embryos, developmental Carnegie stages (CS) and age in postovulatory days of investigated human and rat embryos

\begin{tabular}{llccc}
\hline & CS & $\mathbf{1 4}$ & $\mathbf{1 8}$ & $\mathbf{2 0}$ \\
\hline $\begin{array}{l}\text { Human } \\
\text { embryos }\end{array}$ & Number of embryos & 7 & 5 & 10 \\
& Developmental days & $31-35$ & $44-48$ & $51-53$ \\
\hline Rat & Number of embryos & 7 & 5 & 10 \\
embryos & Developmental days & 13 & 15 & 18 \\
\hline
\end{tabular}

The table shows an approximate comparison of days of development in human and rat embryos based upon Carnegie staging.

and incubated overnight at $4^{\circ} \mathrm{C}$ with the primary antibody: rabbit polyclonal antibody to BMP-2 (Abcam, Cambridge, UK, diluted 1:250) or rabbit polyclonal antibody to BMP-4 (Abcam, Cambridge, UK, diluted 1:100). The next day, sections were washed in PBS and incubated with the secondary antibody (VECTASTAIN ABC Universal Kit, Burlingame, USA) for $30 \mathrm{~min}$ at room temperature. Peroxidatic activity was detected with 3.3'-diaminobenzidine (DAB; Vector, Burlingame, USA) and sections were counterstained with haematoxylin. BMP-2 and BMP-4 labelling was expressed by a subjective scale ranging from 0 to $3(0-$ no staining, 1 - weak staining, 2 - moderate staining, 3 - strong staining). Immunohistochemistry negative controls were performed by omitting the primary antibody. BMP-2 and BMP-4 immunohistochemical staining was evaluated in the developing spinal cord separately in its dorsal and ventral parts. Also, staining was evaluated in the adjacent non-neural ectoderm. Two independent observers evaluated the intensity staining; the mean of estimations was calculated and used for statistical analysis. The data were analysed by Kruskal-Wallis test (nonparametric ANOVA). The level of significance was set at $p<0.05$.

\section{RESULTS}

Expression of BMP-2 and BMP-4, as determined by immunohistochemistry, was observed in the spinal cord at all developmental stages analysed in this study (CS 14, CS 18 and CS 20). BMP-2 signal in the developing spinal cord was found to be stronger compared to BMP-4 both in human and rat embryos (Table 2, Figs. 1-4). BMP-2 and BMP-4 exhibited similar immunolocalisation and expression patterns there was slight, statistically insignificant tendency for expression to decline at later stages of development. However, the developing spinal cord of rat embryos 
Table 2. Immunohistochemical estimation of bone morphogenetic proteins (BMP)-2 and BMP-4 in the spinal cord and non-neural ectoderm of the human and rat embryos at different developmental stages

\begin{tabular}{|c|c|c|c|c|c|c|c|}
\hline & & \multicolumn{3}{|c|}{ BMP-2 } & \multicolumn{3}{|c|}{ BMP-4 } \\
\hline & & CS 14 & CS 18 & CS 20 & CS 14 & CS 18 & CS 20 \\
\hline \multirow{3}{*}{$\begin{array}{l}\text { Human } \\
\text { embryos }\end{array}$} & $\mathrm{DP}$ & $2.4 \pm 0.06^{a, g}$ & $2.3 \pm 0.04^{\mathrm{b}, \mathrm{h}}$ & $2.2 \pm 0.09^{c, i}$ & $2.2 \pm 0.03^{\mathrm{d}, j}$ & $1.9 \pm 0.10^{e, k}$ & $1.9 \pm 0.10^{f, l}$ \\
\hline & VP & $1.8 \pm 006^{a}$ & $1.6 \pm 0.05^{b}$ & $1.8 \pm 0.10^{c}$ & $1.0 \pm 0.05^{d}$ & $1.0 \pm 0.10^{e}$ & $1.0 \pm 0.03^{f}$ \\
\hline & NE & $2.6 \pm 0.08^{m, r}$ & $2.3 \pm 0.14^{n}$ & $1.9 \pm 0.03^{r}$ & $1.7 \pm 0.03^{\circ}$ & $1.8 \pm 0.08^{p}$ & $1.4 \pm 0.07$ \\
\hline \multirow{3}{*}{$\begin{array}{l}\text { Rat } \\
\text { embryos }\end{array}$} & $\mathrm{DP}$ & $1.6 \pm 0.06^{g}$ & $1.6 \pm 0.10^{h}$ & $1.4 \pm 0.05^{i}$ & $1.3 \pm 0.08^{j}$ & $1.2 \pm 0.06^{k}$ & $1.2 \pm 0.08^{\prime}$ \\
\hline & VP & $1.0 \pm 0.04$ & $1.1 \pm 0.05$ & $0.9 \pm 0.05$ & $0.8 \pm 0.06$ & $0.8 \pm 0.07$ & $0.7 \pm 0.08$ \\
\hline & $\mathrm{NE}$ & $1.8 \pm 0.04^{\mathrm{m}}$ & $1.4 \pm 0.12^{n, s}$ & $2.1 \pm 0.05^{s}$ & $0.9 \pm 0.12^{0, t}$ & $1.0 \pm 0.06^{p}$ & $1.5 \pm 0.11^{\mathrm{t}}$ \\
\hline
\end{tabular}

CS — Carnegie stages; DP — dorsal part of the developing spinal cord; VP — ventral part of the developing spinal cord; NE — non-neural ectoderm. Immunostaining intensity was graded from weak (1) to strong (3), the data are presented as mean \pm SEM. Significant differences $(p<0.05)$ in staining intensity between groups are indicated with the same letter

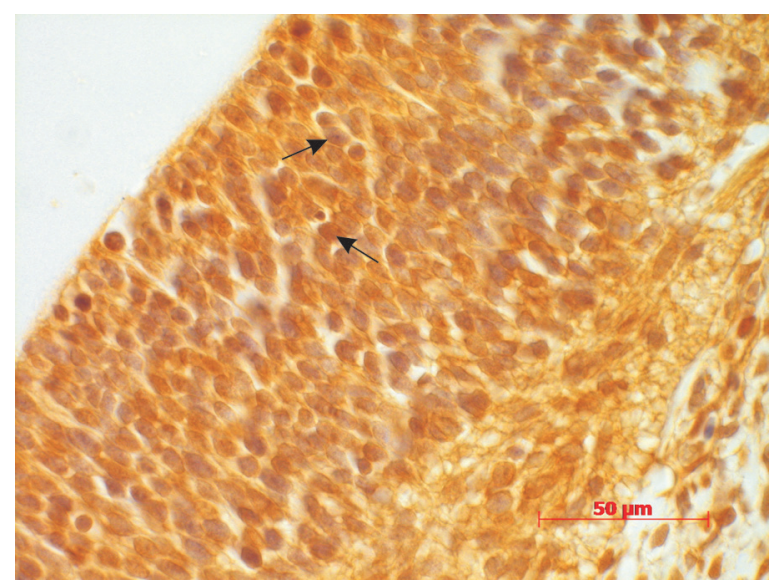

Figure 1. Transversal section of the developing spinal cord of human embryo at Carnegie stage 18. Arrows indicate stronger expression of the bone morphogenetic protein-2 in (BMP-2) neuronal cells.

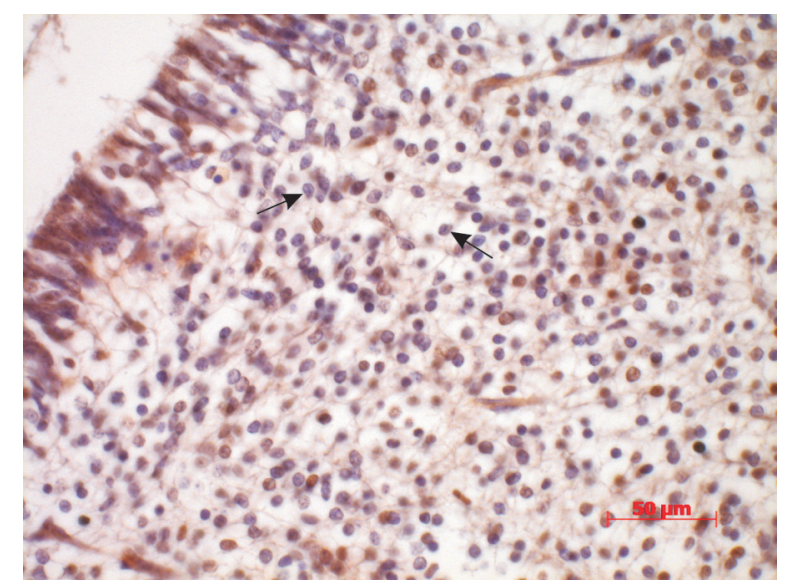

Figure 3. Transversal section of the developing spinal cord of rat embryo at Carnegie stage 18. Arrows indicate moderate expression of bone morphogenetic protein-2 in (BMP-2) neuronal cells.

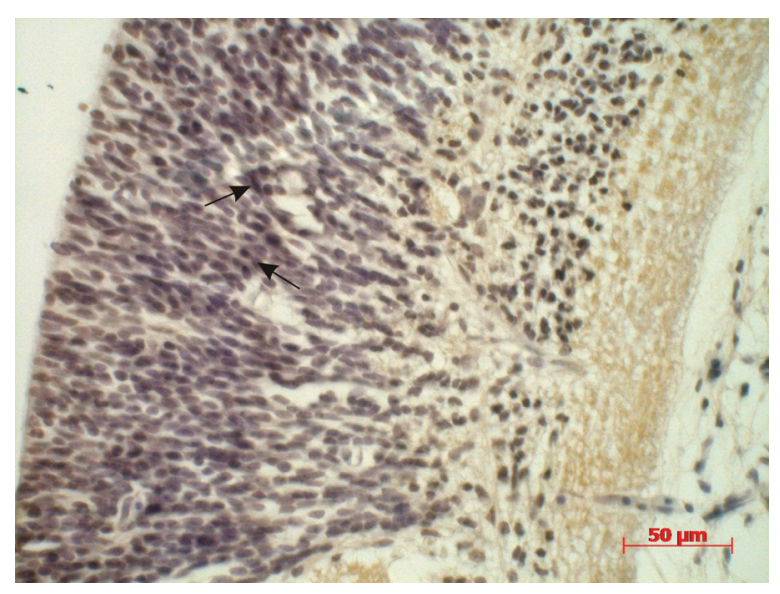

Figure 2. Transversal section of the developing spinal cord of human embryo at Carnegie stage 18. Arrows indicate weaker expression of the bone morphogenetic protein-4 (BMP-4) in neuronal cells.

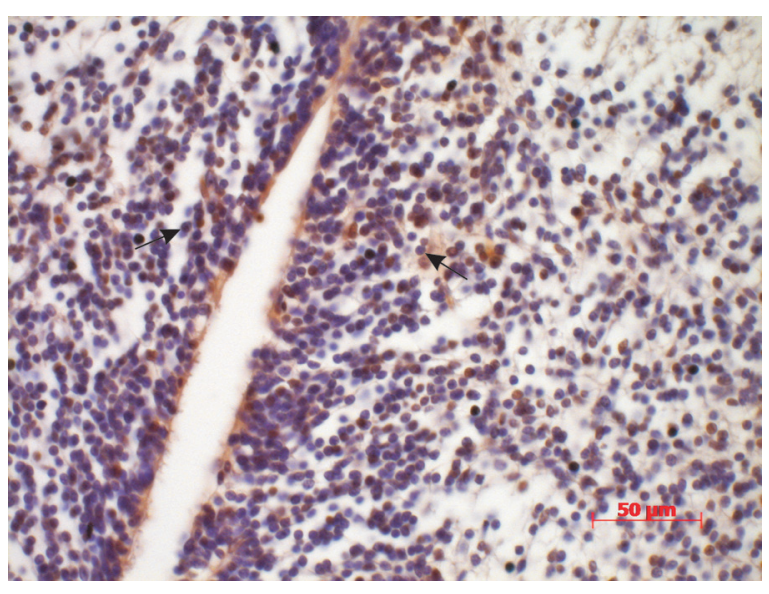

Figure 4. Transversal section of the developing spinal cord of rat embryo at Carnegie stage 18. Arrows indicate weaker expression of bone morphogenetic protein-4 (BMP-4) in neuronal cells. 


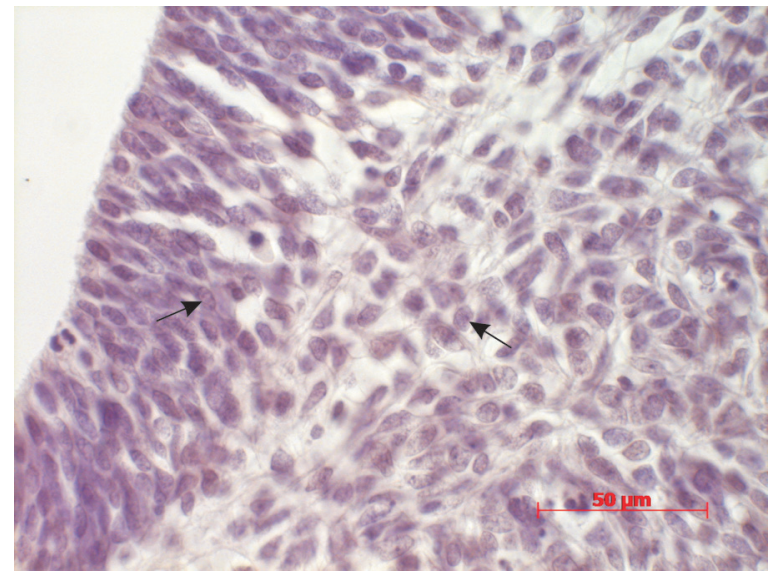

Figure 5. Transversal section of the developing spinal cord of rat embryos at Carnegie stage 20. Arrows indicate very weak expression of bone morphogenetic protein-4 (BMP-4) in neuronal cells.

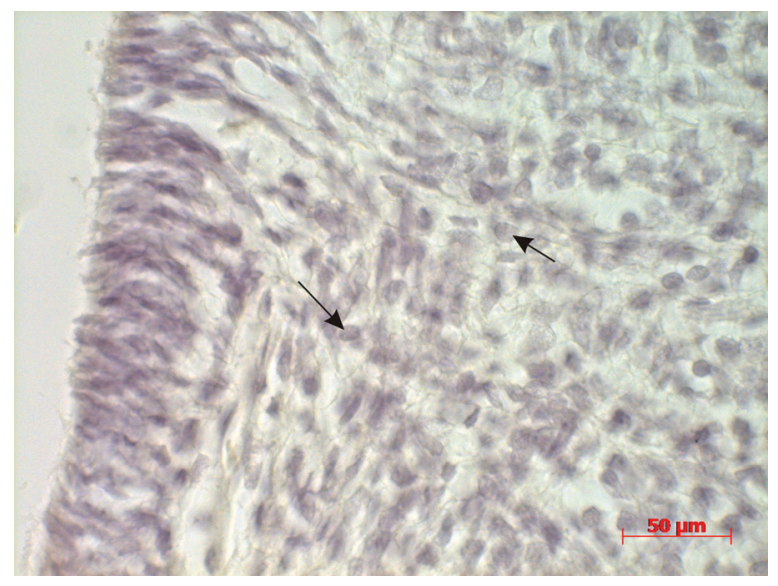

Figure 6. Transversal section of the developing spinal cord of human embryo at Carnegie stage 18. Negative control, arrows indicate no staining intensity in neuronal cells.

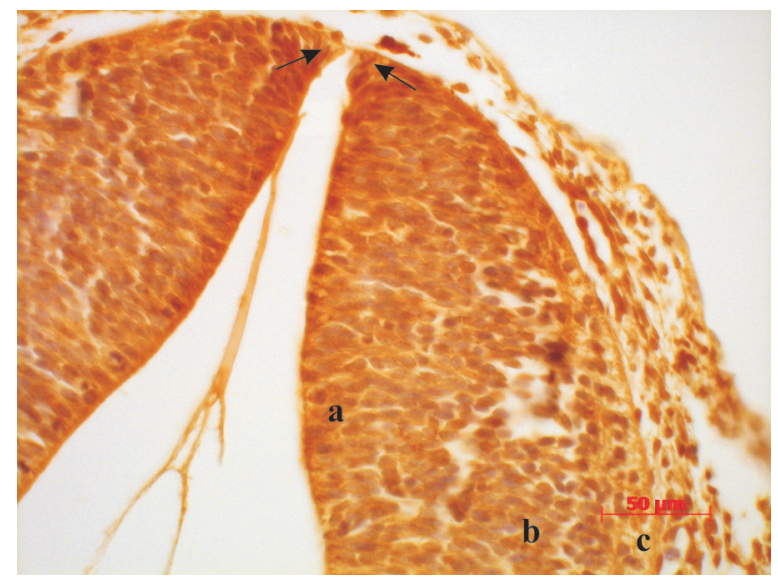

Figure 7. Transversal section of the dorsal part of developing spinal cord of human embryo at Carnegie stage 14. Expression of bone morphogenetic protein-4 (BMP-4) in: a - ventricular layer; $b$ mantel layer; c - marginal layer; arrows indicate roof plate cells.

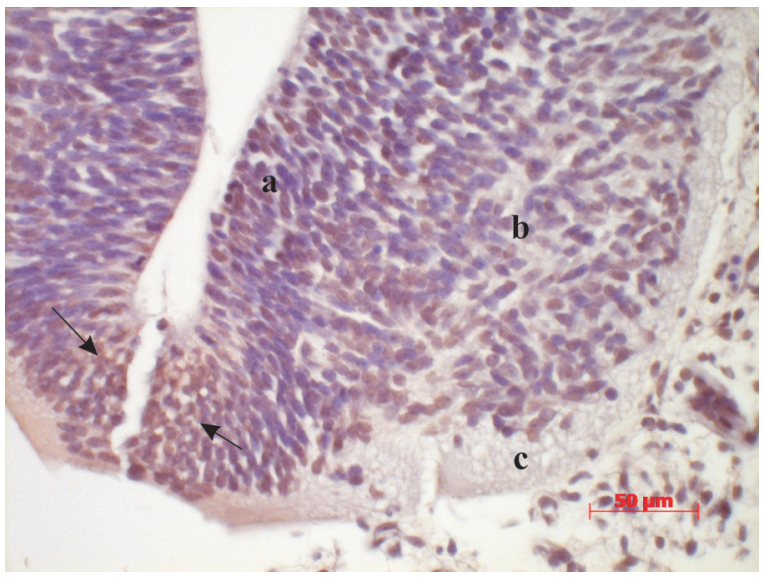

Figure 8. Transversal section of the ventral part of developing spinal cord of human embryo at Carnegie stage 14. Expression of bone morphogenetic protein-4 (BMP-4) in: $a$ - ventricular layer; $b$ mantel layer; c - marginal layer; arrows indicate floor plate cells.

showed weaker immunostaining of BMP-s (Figs. 3, 4) compared to human embryos (Figs. 1, 2; Table 2). Especially weak staining intensity of BMP-4 was detected in the rat embryos at CS 20 (Table 2, Fig. 5). In negative controls where the primary antibodies to BMP-2 or BMP-4 were omitted no staining was detected (Fig. 6).

In the developing spinal cord expression of BMP-2 and BMP-4 was evaluated separately in the roof plate, floor plate and adjacent non-neural ectoderm (Table 2). Statistically significant stronger immunostaining intensity of BMP-s was detected in the dorsal part of both human and rat embryos spinal cord as compared to the ventral parts. At the end of the $5^{\text {th }}$ week of human and 13 days of rat embryos development (CS 14) 3 zones could be distinguish in the wall of the spinal cord: the ventricular, mantel and marginal layer. A comparison of differences between dorsal and ventral part in the spinal cord showed that in human embryos staining intensity of BMP- 2 and BMP-4 was more extensive in 3 layers of the dorsolateral (alar) plates than in ventrolateral (basal) plates (Figs. 7, 8). Similarly, more intensive staining of BMP-2 and BMP-4 was noticed in dorsal parts of the rat spinal cord (Table 2). Also in rat embryos expression of BMP-2 was detected in the ventricular, mantel and basal layers in CS 14-23 of the spinal cord (Fig. 9), but expression of BMP-4 was weaker. Expression of BMP- 2 and BMP- 4 in the roof plate cells was found in both human and rat embryos at all studied CS. We also noticed expression of BMP-2 and BMP-4 in the floor plate (Fig. 8), but expression was found to be less intensive than in the roof plate (Figs. 7, 9). 


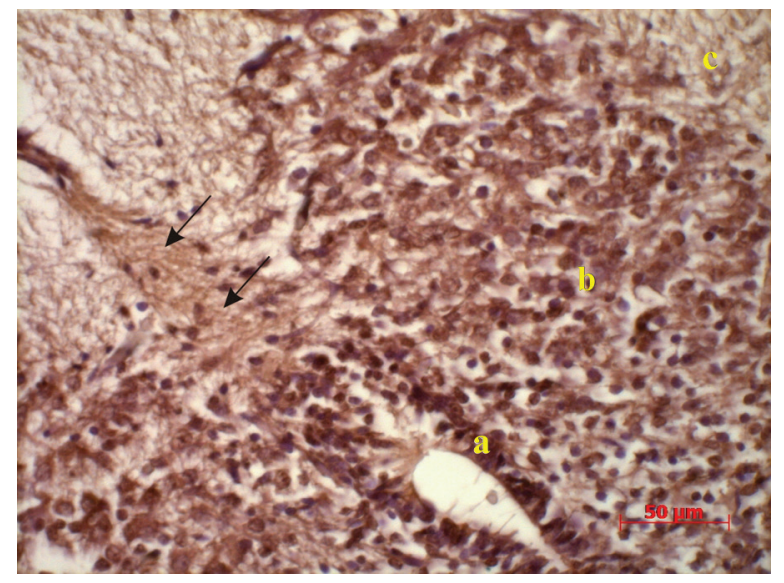

Figure 9. Transversal section of the dorsal part of developing spinal cord of rat embryo at Carnegie stages 20. Expression of bone morphogenetic proteins- 2 in: $a$ - ventricular layer; $b$ - mantel layer; c - marginal layer; arrows indicate roof plate cells.

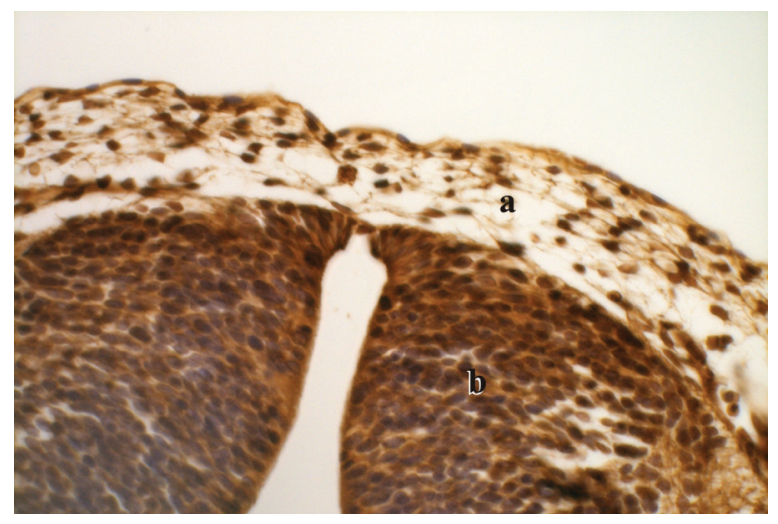

Figure 10. Transversal section of the developing spinal cord and non-neural ectoderm of human embryo at Carnegie stages 18; a expression of bone morphogenetic proteins-2 (BNP-2) in non-neural ectoderm; $b$ - expression of BMP-2 in developing spinal cord.

The expression of BMP-2 and BMP-4 in human and rat embryonic non-neural ectoderm was detected from anterior to posterior level of the forming spinal cord. In the non-neural ectoderm of human embryos BMP-2 and BMP-4 staining was found to be stronger in early stages and weaker in later stages of development (Fig. 10), especially significant higher-level expression of BMP-2 was seen at CS 14 (Table 2). Differently from human embryos, BMP-2 and BMP-4 staining in the non-neural ectoderm of rat embryos was weaker in CS 14-18.

\section{DISCUSSION}

In the present study we compared the expression of BMP-2 and BMP-4 in the developing spinal cord of human and rat embryos. The results, summarised in Table 2, show the temporal pattern of BMP-2 and BMP-4 expression during spinal cord morphogenesis. Our estimations of BMP-2 and BMP-4 immunostaining in the dorsal and ventral parts of the developing spinal cord of human and rat embryos throughout developmental stages CS 14-20 are in general in accordance with data obtained from animal studies. Several investigations in experimental animals, mainly mice and chickens, have demonstrated that the normal development of the spinal cord is tightly regulated by BMP signalling [13]. High levels of BMP signalling set up the most dorsally located precursor cells and lower levels of signalling define ventrally located cells. The regionalisation of the precursor cells leads to the formation of distinct dorsal and ventral neuronal cell types $[6,10]$. In our study stronger immunostaining of BMP-s was seen in the dorsal part of the developing spinal cord: higher expression of BMP-2 and BMP-4 was noted in the ventricular, mantel and marginal layers of the dorsal part in developing spinal cord of both human and rat embryos (Figs. 7, 9), while staining in the ventral part was weaker (Fig. 8). Similar expression gradient of BMP-s has been found in other experimental animals $[6,10,13]$. In our study on human and rat embryos, BMP-2 and BMP-4 expression was also noted in the roof plate (Fig. 7), which is in concordance with studies on mice, where it is demonstrated that BMP-s mediated signals from the roof plate are required for the differentiation of neuronal cells in the dorsal spinal cord [1,2].

Comparing staining estimations of BMP-2 and BMP-4 in the dorsal part of the developing spinal cord there were no significant differences between the consecutive developmental stages (from CS 14 to CS 20) both in human and rat embryos, though slight declining tendency was noted in later developmental stages (Table 2). However, this tendency was more visible in our previous study on neural tubes of human embryos where more earlier developmental stages were investigated, in particular clear differences in BMP-4 immunostaining intensity was noticed with higher level at CS 10 and lower level at CS 20 [11]. This seems to support the idea that the roles of BMP-s are the most significant after the neural tube closing when the differentiation of the neural tube begins [10].

Although resembling descriptions in experimental animals, human BMP-2 and BMP-4 immunostaining in the developing spinal cord displayed slight diffe- 
rences in their expression pattern when compared to rat embryos. It refers to the higher expression of both proteins in the dorsal part of the developing spinal cord of human embryos. Also, when in human embryos BMP-2 and BMP-4 immunostaining in the non-neural ectoderm declined throughout the consecutive developmental stages, then in rat embryos there was an opposite tendency as the expression of BMP-s increased from CS 14 to CS 20 (Table 2). There is evidence from studies on chick spinal cord that BMP-2 and BMP-4 produced by dorsal non-neural ectoderm (surface ectoderm) function as dorsalizing signals to pattern adjacent neuroectoderm [7-9, 14]. The strong to moderate level of BMP- 2 and BMP-4 protein expression in the non-neural ectoderm seen in our study suggest that BMP-s could likely have similar involvement in the dorsal neural tube development of the human embryos, especially in the early stages of development (Table 2).

\section{CONCLUSIONS}

It can be concluded that protein expressions of BMP-2 and BMP-4 in the developing spinal cord of human embryos during the studied CS 14, CS 18 and CS 20 have similar spatial and temporal pattern to the corresponding stages of rat embryos. Similarly higher expression of BMP-s in the dorsal and lower expression in the ventral part of the developing spinal cord was seen in both human and rat embryos thus resembling the situation described in other vertebrates. However, in the non-neural ectoderm adjacent to the developing spinal cord staining of BMP-s in the human embryos seemed to have a tendency to decrease from earlier to later developmental stages, while in rat embryos the situation was in opposite as the staining of BMP-s was found to be stronger in the last studied developmental stage compared to the earlier stages.

\section{ACKNOWLEDGEMENTS}

The study has been partly funded by the target financed project No. $0180012 \mathrm{~s} 11$ of the Estonian Ministry of Education and Research. The help in the process of collection of human embryos by $\operatorname{Dr} A$. Sizarov and rat embryos by Prof. G. Selstam from the University of Umea (Sweden) is greatly acknowledged.

\section{REFERENCES}

1. Chizhikov VV, Millen KJ (2004) Mechanism of roof plate in vertebrate CNS. Nat Rev Neurosci, 5: 808-812.

2. Chizhikov, VV, Millen KJ (2005) Roof plate-dependent patterning of the vertebrate dorsal central nervous system. Dev Biol, 277: 287-295.

3. Faure S, de Santa Barbara P, Roberts DJ, Whitman M (2002) Endogenous patterns of BMP signaling during early chick development. Dev Biol, 244: 44-65.

4. Hogan BL (1996) Bone morphogenetic proteins: multifunctional regulators of vertebrate development. Genes Dev, 10: 1580-1594.

5. Hu Q, Ueno N, Behringer RR (2004) Restriction of BMP-4 activity domains in the developing neural tube of the mouse embryo. EMBO, 5: 734-739.

6. Jessell TM (2000) Neuronal specification in the spinal cord: inductive signals and transcriptional codes. Genetics, 1:20-29.

7. Lee KJ, Mendelsohn M, Jessell TM (1998) Neuronal patterning by BMP-s: a requirement for GDF7 in the generation of a discrete class of commissural interneurons in the mouse spinal cord. Genes Development, 12: 3394-3407.

8. Liem KF, Tremml G, Jessell TM (1997) A role for the roof plate and its resident TGFbeta-related proteins in neuronal patterning in the dorsal spinal cord. Cell, 91: 127-138.

9. Liem KF, Tremml G, Roelink H, Jessell TM (1995) Dorsal differentiation of neural plate cells induced by BMP-mediated signals from epidermal ectoderm. Cell, 82: 969-979.

10. Liu A, Niswander LA (2005) Bone morphogenetic protein signaling and vertebrate nervous system development. Neuroscience, 6: 945-954.

11. Namm A, Arend A, Aunapuu M (2013) Immuohistochemical detection of BMP-2 and BMP-4 in the developing neural tube and spinal cord of human embryos. Int J Morphol, 31: 473-479.

12. O'Rahilly R, Müller F (1987) Developmental stages in human embryos. Carnegie Institution of Washington, Washington, DC, pp. 1-306.

13. Stern CD (2005) Neural induction: old problem, new findings, yet more questions. Development, 132: 2007-2021.

14. Timmer JR, Wrang C, Niswander L (2002) BMP signaling patterns the dorsal and intermediate neural tube via regulation of homeobox and helix-loop-helix transcription factors. Development, 129: 2459-2472.

15. Ulloa F, Briscoe BJ (2007) Morphogenesis and the control of cell proliferation and patterning in the spinal cord. Cell Cycle, 6: 2640-2649.

16. Wilson L, Maden M (2005) The mechanisms of dorsoventral patterning in the vertebrate neural tube. Dev Biol, 282: 1-13. 